\title{
Early social experience influences the development of stereotypic behaviour in captive-born striped mice Rhabdomys
}

\author{
Megan Anne Jones ${ }^{\mathrm{a}, *}$, Georgia Mason ${ }^{\mathrm{b}}$, Neville Pillay ${ }^{\mathrm{a}}$

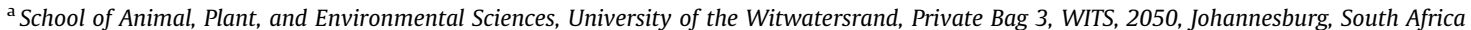 \\ ${ }^{\mathrm{b}}$ Department of Animal and Poultry Sciences, University of Guelph, 50 Stone Road East, Guelph, Ontario, Canada, N1G 2W1
}

A R T I C L E I N F O

Article history:

Accepted 17 December 2009

Available online 21 January 2010

\section{Keywords:}

Biparental care

Early experience

Paternal care

Social enrichment

Stereotypic behaviour

Striped mouse

Weaning age

\begin{abstract}
A B S T R A C T
The early life experience of captive animals, in combination with their genetic inheritance, can predispose or promote the development of stereotypic behaviour in later life. To investigate the early social effects of weaning age and the presence/absence of the father on the development of stereotypic behaviour in adulthood, we retrospectively analysed data from our captive colony of striped mice, Rhabdomys. In the first analysis, pairs of young from each litter were respectively weaned on postnatal days 12 (4 days before natural weaning), 16 , or 20 , and the incidence of stereotypic behaviour recorded between days 50 and 60. Early weaning (day 12) was associated with a significantly higher incidence of stereotypic behaviour than later weaning (day 16 or 20). In the second analysis, pups were raised with either the father present (biparental care) or absent (uniparental care). Biparentally reared animals showed significantly less stereotypic behaviour as adults than individuals reared by their mother alone. In both analyses, young from stereotypic mothers showed a higher incidence of stereotypic behaviour than offspring from non-stereotypic dams, with experiential and genetic effects combining to influence the adult phenotype. Together, these data indicate that the early social environment influences the development of stereotypic behaviour in adulthood, and suggest directions for future research into the mechanisms of the epigenetic effects of early social experience on the development of stereotypic behaviour, such as whether amounts of parental care received per pup cause these effects, and whether changes in stereotypic behaviour are paralleled by changes in stress responsiveness.
\end{abstract}

(c) 2010 Elsevier B.V. All rights reserved.

\section{Introduction}

Early life experience, in concert with genetic inheritance, has profound and enduring effects on the organisation of the central nervous system (CNS) and thus an animal's behavioural, psychological, and physiological responses to the environment (Laviola and Terranova, 1998; Caldji et al., 2000; Gilmer and McKinney, 2003). Whereas free-ranging young mammals usually have extended physical and social contact with their mother, father and/or family group,

\footnotetext{
* Corresponding author. Tel.: +27 11717 6400; fax: +27 114031429 E-mail address: megan.jones@icon.co.za (M.A. Jones).
}

captivity is often associated with both parental and social deprivation (Latham and Mason, 2008; Newberry and Swanson, 2008): individuals typically experience an earlier and more abrupt weaning than is natural, and are often not raised in their species-typical social groupings which might include the father (e.g. biparental care in some rodent species; Dewsbury, 1985) or the larger family unit (e.g. communal nesting in mice, Mus musculus; Hayes, 2002). Because the epigenetic effects of early social deprivation modify stress responsiveness in later life (Caldji et al., 2000) these effects can, in the long-term, affect the adjustment of animals to the captive environment.

The adverse effects of premature weaning on general behaviour are well characterised in rodents (Laviola and 
Terranova, 1998; Kikusui et al., 2008). Early weaning results in both deprivation of maternal care and separation from the mother before adequate developmental maturity. This deprivation induces immediate neuroendocrine and behavioural stress responses, causing long-lasting effects on brain and behaviour, in particular stress reactivity (Kikusui et al., 2008). In the few existing studies investigating the association between weaning age and the development of abnormal behaviour, premature weaning has been linked to the later development of stereotypic behaviour, repetitive behaviour that is caused by frustrated motivation to perform (or receive, in the case of infants) species-typical behaviour and/or from the chronic impact of deprived conditions on CNS development and function (Latham and Mason, 2008). For instance, laboratory mice weaned at 17 days showed increased rearing (upright standing on the hind legs, often associated with single vertical jumps at the cage wall) shortly after weaning, and performed more stereotypic behaviour (bar-biting) in adulthood than mice weaned 4 days later at 21 days (Würbel and Stauffacher, 1997). In farmed mink (Mustela vison), whilst there were no observable effects of weaning age on activity immediately post-weaning, the early weaned mink in one study showed increased tail-biting several months later (Mason, 1995) and, in another study, higher levels of stereotypic behaviour (Jeppesen et al., 2000). These, often initially latent, effects of early weaning suggest early but pervasive changes in motivational state or in the control of behaviour (Latham and Mason, 2008), although these changes have yet to be assessed directly. In contrast with the effects of premature weaning, delayed weaning is associated with greater exploration in adulthood (Adriani and Laviola, 2002) and, in certain rearing environments, with reduced anxiety and abnormal behaviour (stereotypic behaviour and barberingself-removal of hair, or removal of hair by a cagemate; Bechard et al., submitted for publication).

Naturally occurring variations in maternal care (e.g. the amount of licking and grooming provided to pups) influence neuroendocrine development, and consequently adult behaviour and effects mediated through epigenetic modification of gene expression in the brain (Caldji et al., 2000; Champagne and Curley, 2009). However, little is known about the effects of paternal care in biparental species (Bredy et al., 2007). The best characterised model thus far of biparental care is the California mouse (Peromyscus californicus), in which biparental rearing results in pups receiving more licking and grooming than if only the mother were present (Wright and Brown, 2002; Bredy et al., 2004). In this species, it has also been shown that the presence of the father affects the development of aggression (Frazier et al., 2006; Bester-Meredith and Marler, 2007) and cognition (novel object recognition; Bredy et al., 2004). To our knowledge, however, the effect of biparental care on stress responsiveness has not been tested, nor the link assessed between variations in maternal or paternal care and the development of stereotypic behaviour.

In this study, we investigated the roles of (1) weaning age and (2) the absence/presence of the father (uni- or biparental care) on the development of stereotypic behaviour by retrospectively analysing data from our captive colony of striped mice, Rhabdomys, a species showing highly developed paternal care (Schradin and Pillay, 2003). In the first analysis, we assessed the effect of weaning age (days 12, 16, or 20) on expression of stereotypic behaviour in adult striped mice (50-60 days). In the second analysis, we compared the incidence of stereotypic behaviour in adult striped mice that were raised by the mother alone (uniparental care) or by both parents (biparental care). In both analyses, because stereotypic behaviour is known to be genetically (but not socially) transmitted in striped mice (Schwaibold and Pillay, 2001; Jones et al., 2008), we controlled for the stereotypic status (stereotypic or non-stereotypic) of the parents, and also investigated the potential interaction of genotype with the respective early social experiences. We predicted that early weaning would predispose young to the development of stereotypic behaviour, whereas biparental care would protect against its development.

\section{General approach}

\subsection{Housing}

All striped mice were housed in the Milner Park Animal Unit at the University of the Witwatersrand, under partially controlled environmental conditions (14L:10D, lights on at 05:00 h; $20-24{ }^{\circ} \mathrm{C} ; 30-60 \%$ relative humidity). Individuals were kept singly, in pairs, or in same-sex sibling groups in standard Labotec cages $(300 \mathrm{~mm} \times 200 \mathrm{~mm} \times 150 \mathrm{~mm}$ ), containing $\sim 2 \mathrm{~cm}$ woodshavings as bedding and a handful of hay as nesting material. Epol ${ }^{\circledR}$ mouse cubes and water were provided ad libitum. The diet was supplemented twice weekly with a small amount $( \pm 5 \mathrm{~g})$ of parrot seed mix.

\subsection{Approach to analysis}

This study is based on retrospective analyses of data collected from NP's striped mouse colonies between 1997 and 2005. Whilst care has been taken to exclude potential biases through careful a priori selection of data and/or to account for biases in the data sets using statistical methods (e.g. we factor in the stereotypic status of the parents), some minor confounds remain in the analyses, which we acknowledge, and discuss in the respective discussion sections for each analysis (below).

\subsection{Ethical note}

Animal use and care, in the relatively barren conditions described above, conformed to our institution's ethical guidelines at the time when the research was conducted. We have subsequently modified our animal care protocols to mandate basic enrichment in order to enhance the welfare of the striped mice and also to reduce the incidence of stereotypic behaviour.

\section{Weaning age}

\subsection{Methods and materials}

\subsubsection{Procedure}

Juvenile offspring from G1 to G4 breeding pairs that produced six pups (the mean litter size for captive 
Rhabdomys is $7.2 \pm 1.8$; Pillay, 2000) were included in a socio-ecological study investigating the effect of weaning age on life history strategy (Pillay, unpublished data). Founder stock was from a Highveld grassland locality $\left(25^{\circ} 40^{\prime} \mathrm{S}\right.$; $28^{\circ} 30^{\prime} \mathrm{E}$ ). Fathers were separated from the mothers before parturition to prevent post-partum insemination and a second pregnancy. In total, 20 litters were used in the analysis: in 10 of these litters both parents were nonstereotypic, and in 10 litters the mother was stereotypic and the father non-stereotypic; Jones et al. (2008) describe in detail how the stereotypic status of the striped mice was established. In each litter, pairs of offspring were randomly selected for weaning (defined here as physical separation from the mother) at days 12 (2/litter), 16 (2/litter), or 20 (2/ litter), with weaning at day 16 corresponding with the typical weaning age for striped mice in the field (Brooks, 1982). Within litters, sex ratio was not always equally balanced but, across litters, males and females were equally represented in each treatment group. After weaning, striped mice were housed in sibling pairs until day 60. Stereotypic status (stereotypic; non-stereotypic) was assessed between days 50 and 60 using daily direct observations, for $15 \mathrm{~min}$, over five non-consecutive days (Jones et al., 2008), and the proportion calculated of offspring in each sibling pair developing stereotypic behaviour. Stereotypic behaviours observed were all locomotor, and included circuit-running, somersaulting, cage-climbing, and jack-hammering. These forms of abnormal behaviour were regarded as stereotypic if the behaviour comprised at least three repetitions in succession (see Jones et al., 2008). Mass at weaning was also recorded but, because striped mice in each sibling pair were not individually marked at weaning, we cannot link individual weaning mass to stereotypic status in adulthood.

\subsubsection{Data analysis}

To assess the effect of weaning age on the development of stereotypic behaviour, we used a general linear model (GLM) with maternal stereotypic status as a categorical predictor, weaning age as the repeated measure, and the proportion of offspring (in each of the three paired sibling pairs, from each of 20 litters) developing stereotypic behaviour as the dependent variable. Proportion data were arcsine square root transformed prior to analysis (Zar, 1996). Tukey post hoc tests were used to identify specific differences when $\alpha \leq 0.05$. All analyses were performed using Statistica 8.0 (Statsoft Inc. 2007). Because mass at weaning can influence the development of stereotypic behaviour (Würbel and Stauffacher, 1997), we also present summary data (mean + SEM) for the averaged mass of individuals within each sibling pair at weaning on days 12 , 16 , and 20 in relation to the range of weaning mass observed in free-living striped mice.

\subsection{Results}

Weaning age predicted stereotypic behaviour development $\left(F_{2,36}=11.26, P<0.001\right)$, with striped mice weaned at 12 days far more likely to develop stereotypic behaviour as mice weaned at either 16 or 20 days $(P<0.001)$ (Fig. 1$)$. Maternal stereotypic status, too, was a significant predictor of offspring stereotypic behaviour development

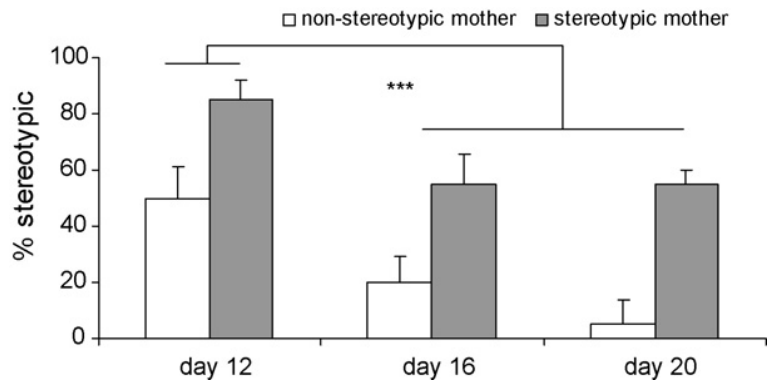

Fig. 1. The incidence of stereotypic behaviour (mean + SEM) in offspring pairs weaned at days 12,16 , or 20 from non-stereotypic (white bars) and stereotypic (grey bars) mothers. At each weaning age, offspring from nonstereotypic mothers were less likely to be stereotypic than were offspring from stereotypic mothers. ${ }^{* *} P<0.001$

$\left(F_{1,18}=24.22, \quad P<0.001\right)$ with offspring of stereotypic mothers more likely to develop stereotypic behaviour than offspring of non-stereotypic mothers (Fig. 1). The interaction between maternal stereotypic status and weaning age was not a significant predictor of the incidence of stereotypic behaviour. As would be expected, mass at weaning was higher in older animals and, for mice weaned at days 16 or 20 , was at least as high as is typically recorded in free-living animals (Fig. 2).

\section{Uniparental v. biparental care}

\subsection{Methods and materials}

\subsubsection{Procedure}

Subjects used in this analysis were offspring from a separate group of G1-G4 captive-born breeding pairs (32 pairs) used as part of various socio-ecological studies investigating, inter alia, the effect of the father on offspring physical development (Schradin and Pillay, 2003; Nel, 2003). The founder stock also originated from a Highveld grassland. Breeding pairs had produced at least one previous litter. Fathers were all non-stereotypic, whereas half of the mothers were stereotypic, and half non-stereotypic. The first litter used for study was raised by both parents (biparental care). Since Rhabdomys show post-partum oestrus, females were inseminated soon after the birth of their first litter. Fathers were separated from the females when the first litter was weaned at 16 days and thus, for the second litter (born 7-11 days after the first litter was weaned), young were raised by the mother alone (uniparental care). After weaning at 16 days, offspring were housed in same-sex sibling groups. Stereotypic status of the offspring (stereotypic; non-stereotypic) was assessed between days 50 and 60, as described for the first analysis, and the proportion calculated of offspring in each litter (male and female combined) developing stereotypic behaviour.

\subsubsection{Data analysis}

To assess the effect of the presence of the father on the development of stereotypic behaviour, we used a GLM with maternal stereotypic status as a categorical predictor, parental care type (uni- v. biparental) as a repeated measure, and the proportion of offspring in each litter 
unon-stereotypic mother $\square$ stereotypic mother

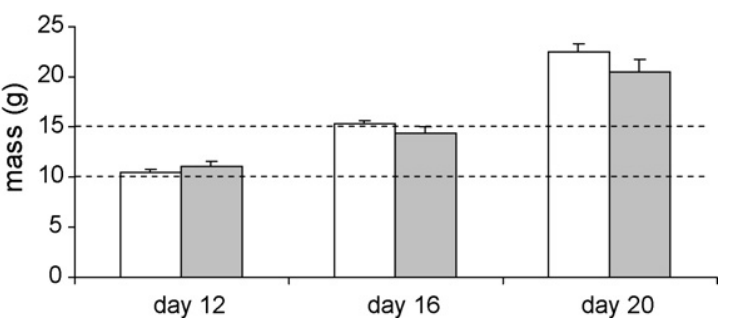

Fig. 2. Mean mass at weaning (+SEM) of offspring pairs weaned at days 12 , 16, or 20 from non-stereotypic (white bars) and stereotypic (grey bars) mothers. The dashed lines indicate weaning mass range for free-living striped mice ( day 16; Brooks, 1982).

口non-stereotypic mothera stereotypic mother

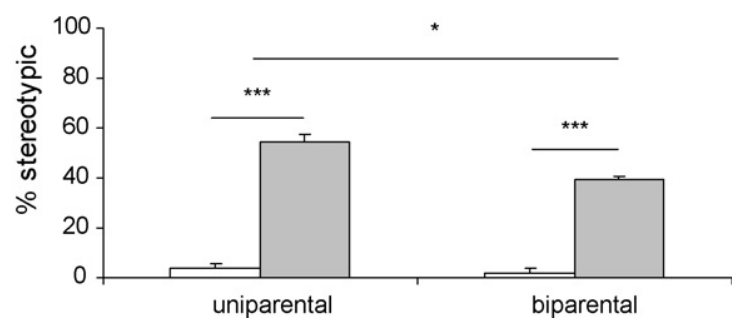

Fig. 3. The incidence of stereotypic behaviour ( mean + SEM) in litters from a stereotypic or non-stereotypic mother receiving either uniparental or biparental care. In each parental care type, offspring from non-stereotypic mothers were less likely to be stereotypic than were offspring from stereotypic mothers. ${ }^{*} P<0.05$; ${ }^{* * *} P<0.001$.

( $n=32$ paired litters in each parental care type) developing stereotypic behaviour as the dependent variable. Data were arcsine square root transformed prior to analysis (Zar, 1996). Tukey post hoc tests were used to identify specific differences when $\alpha \leq 0.05$. All analyses were performed using Statistica 8.0 (Statsoft Inc. 2007).

\subsection{Results}

Parental care type significantly predicted stereotypic behaviour development $\left(F_{1,30}=5.14, P=0.03\right)$, with young raised by both parents showing a lower incidence of stereotypic behaviour than young raised by mothers alone (Fig. 3). Maternal stereotypic status was again a significant predictor of offspring stereotypic behaviour development $\left(F_{1,30}=326.23, P<0.001\right)$ with offspring of stereotypic mothers more likely to develop stereotypic behaviour than offspring of non-stereotypic mothers. As was the case with weaning age, there was no significant interaction between maternal stereotypic status and parental care type.

\section{Discussion}

The results from our retrospective analyses support our prediction that early social experiences, specifically those relating to weaning age and the absence or presence of the father, have significant effects on the development of stereotypic behaviour in later life. They also show that in striped mice, experiential and genetic effects seem to combine in an apparently additive way to produce the stereotypic phenotype.

\subsection{Weaning age}

As predicted, early weaning at day 12 predisposed striped mice to the development of stereotypic behaviour. Although these early weaned striped mice were able to feed on solid food and move independently from their mother, in both the field and in captivity they would have continued to suckle and be in close contact with their mothers until at least 16 days (Brooks, 1982). We had expected that slightly delayed weaning (days 20 v. 16) would protect against stereotypy development, given that pups in nature usually experience some interaction with their mothers (if not suckling) after their initial dispersal at 16 days (Willan and Meester, 1989), but this was not supported in our analysis. Future work should investigate whether (1) a more extended period of contact between mothers and pups is necessary (which more closely approximates the 25-day contact period in the wild before the next litter is born, after which contact is minimal despite juveniles remaining in the natal territory; Willan and Meester, 1989) to protect striped mice from the development of stereotypic behaviour; and/or (2) delayed weaning might be of less benefit to offspring when it extends beyond the natural weaning period for the species (c.f. delaying the weaning age in a standard mouse facility in which separation is prior to natural weaning and dispersal age; Bechard et al., submitted for publication).

In laboratory mice, Würbel and Stauffacher (1997) demonstrated that not only weaning age, but also developmental stage at weaning, is a predictor of stereotypic behaviour development, with mice of lighter mass more likely to develop stereotypic behaviour than heavier animals-potentially because the stress involved in breaking the maternal bond is increased in those individuals more likely to experience a greater fitness cost. Indeed, young striped mice in our study on day 12 were, as expected, lighter than at day 16 , and lighter at day 16 than at day 20. Whilst it is possible that in striped mice maternal deprivation per se may play a greater role in stereotypic behaviour development than developmental immaturity (c.f. Latham and Mason, 2008), especially because laboratory conditions promote more rapid weight gain than in the wild (Pillay, 2000), further work is needed to tease apart the correlated effects of treatment (age at weaning) and body mass by looking at individual differences in mass at weaning and the subsequent development of stereotypic behaviour. In fact, the large mass difference in striped mice weaned at days 16 and 20, yet their similar susceptibility to the development of stereotypic behaviour, suggests that weaning mass does not determine vulnerability to stereotypic behaviour in a simple linear way.

A confound in this analysis is that striped mice from the same litter were weaned at different ages (see also Cook, 1999; Kikusui et al., 2007). Whilst such a method is desirable because it reduces the number of animals used, it confounds weaning age with a reduction in litter size. Future work is thus also required to replicate this finding 
when isolating the effect of weaning age from that of reduced litter size and the loss of siblings-especially since there is a known link in this species between stereotypic behaviour and large litter sizes (Jones et al., in press).

\subsection{Uniparental $v$. biparental care}

To our knowledge, this is the first study to suggest an effect of biparental care on the development of stereotypic behaviour. Whilst subjects used in this study originated from a population (Highveld grasslands) in which paternal care does not occur in nature, this population shows highly developed paternal care in the laboratory (Schradin and Pillay, 2003). Biparentally reared striped mouse pups thus likely receive greater parental contact, including licking and grooming, than pups would receive from their mothers alone, although they do not, in the laboratory, show more rapid growth or development (Schradin and Pillay, 2003; Rymer, 2009). Since greater maternal investment is associated with reduced stress responsiveness in offspring (Caldji et al., 2000), the provision of higher levels of total parental care thus seems a likely mechanism through which the incidence of stereotypic behaviour in striped mice is reduced in litters raised by both parents.

Two confounding variables, because of the unbalanced nature of the experimental design, weaken our interpretation of these results: (1) Females were pregnant whilst rearing the first, but not second litter: pregnancy whilst lactating can sometimes reduce maternal care (e.g. in mice, König and Markl, 1987) which we would expect to increase the incidence of stereotypic behaviour, and which would thus have reduced, and not inflated, the significance of our result; and (2) mothers in their second parity were subject to removal of the father before birth, which could potentially induce stress (although this is less likely to occur in striped mice than in monogamous species as free-ranging individuals do not establish pair bonds). Nonetheless, future work should attempt to confirm the effect of paternal care whilst controlling for pregnancy status and the potential stress of separation.

More generally, we need now to investigate the ways in which life experiences either predispose or promote stereotypic behaviour in later life. Candidate mechanisms for such epigenetic influences include (1) dysregulation of behavioural control as a consequence of adverse events during early experience canalising dysfunctional brain development (e.g. Lewis et al., 2006), and/or (2) increasing stress responsiveness and/or the performance of source behaviours as a result of the lack of paternal input/early separation from the mother (e.g. Würbel and Stauffacher, 1997), mechanisms potentially mediated by the quality or quantity of parental contact received by pups. The stressrelated or welfare correlates of these early social experiences particularly merit future research since the effects of these events do not necessarily co-vary with the expression of stereotypic behaviour (Mason and Latham, 2004) but, similarly to stereotypic behaviour, have bearings on animal well-being and the validity of research (Jones et al., in preparation).

\section{Conclusion}

Our findings add to evidence that early social experience can have lasting effects on adult abnormal behaviour, and suggest that Rhabdomys provide a promising model for investigating the effects of weaning age and paternal care on the development of stereotypic behaviour. In this species, genetic predisposition combines with early social experience. We suggest that future prospective studies control for the confounding issues discussed above and further investigate the age when captive striped mice would chose voluntarily to leave the nest, in the presence and the absence of the father, and in relation to developmental stage, and whether the presence of the father would be protective against the development of stereotypic behaviour in early weaned striped mice. Future work should also investigate the mechanisms through which weaning age and paternal care (and/or their correlates in the experiments reported here) modify the development of stereotypic behaviour, including whether amounts of parental care received per pup mediate these effects, and whether increases in stereotypic behaviour are paralleled by increases in stress responsiveness and/or brain dysfunction.

\section{Acknowledgements}

Funding was provided by the National Research Foundation (NRF; grant number: 2069110) and the University of the Witwatersrand. MJ was partially supported whilst writing this paper by an NRF Scarce Skills Scholarship. GM was funded by an NSERC Canada Research Chair. Appreciative thanks to Lis Jones for her helpful proofreading and to two anonymous reviewers for their valuable comments on an earlier draft of this manuscript. Approval for these studies was provided by the Animal Ethics Screening Committee of the University of the Witwatersrand.

\section{References}

Adriani, W., Laviola, G., 2002. Spontaneous novelty seeking and amphetamine-induced conditioning and sensitization in adult mice: evidence of dissociation as a function of age at weaning. Neuropsychopharmacology 27, 225-236.

Bechard, A., Roder, J., Nicholson, A., Mason, G., submitted for publication. (Laboratory Animals). The effects of delayed weaning on the behaviour of laboratory mice.

Bester-Meredith, J.K., Marler, C.A., 2007. Social experience during development and female offspring aggression in Peromyscus mice. Ethology $113,889-900$

Bredy, T.W., Brown, R.E., Meaney, M.J., 2007. Effect of resource availability on biparental care, and offspring neural and behavioural development in the California mouse (Peromyscus californicus). Eur. J. Neurosci. 25, 567-575.

Bredy, T.W., Lee, A.W., Meaney, M.J., Brown, R.E., 2004. Effect of neonatal handling and paternal care on offspring cognitive development in the monogamous California mouse (Peromyscus californicus). Horm. Behav. 46, 30-38.

Brooks, P.M., 1982. Aspects of the reproduction, growth and development of the four-striped field mouse, Rhabdomys pumilio (Sparrman, 1784). Mammalia 46, 53-64.

Caldji, C., Diorio, J., Meaney, M.J., 2000. Variations in maternal care in infancy regulate the development of stress reactivity. Bio. Psychiatry 48, 1164-1174.

Champagne, F.A., Curley, J.P., 2009. Epigenetic mechanisms mediating the long-term effects of maternal care on development. J. Neurosci. Biobehav. Rev. 33, 593-600. 
Cook, C.J., 1999. Patterns of weaning and adult response to stress. Physiol. Behav. 67, 803-808.

Dewsbury, D.A., 1985. Paternal care in rodents. Am. Zool. 25, 841-852.

Frazier, C.R.M., Trainor, B.C., Cravens, C.J., Whitney, T.K., Marler, C.A., 2006. Paternal behaviour influences development of aggression and vasopressin expression in male California mouse offspring. Horm. Behav. 50, 699-707.

Gilmer, W.S., McKinney, W.T., 2003. Early experience and depressive disorders: human and non-human primate studies. J. Affect. Disorders $75,97-113$.

Hayes, L.D., 2002. To nest communally or not to nest communally: a review of rodent communal nesting and nursing. Anim. Behav. 59, 677-688.

Jeppesen, L.L., Heller, K.E., Damgaard, T., 2000. Effects of early weaning and housing conditions on the development of stereotypies in farmed mink. Appl. Anim. Behav. Sci. 68, 85-92.

Jones, M., van Lierop, M., Pillay, N., 2008. All a mother's fault? Transmission of stereotypy in striped mice Rhabdomys. Appl. Anim. Behav. Sci. $115,82-89$.

Jones, M.A., Mason, G., Pillay, N., in press. Increased reproductive output in stereotypic captive Rhabdomys females: potential implications for captive breeding. Appl. Anim. Behav. Sci., doi:10.1016/j.applanim. 2009.12.013.

Jones, M.A., Mason, G., Pillay, N., in preparation. Causes and consequences of stereotypy in the striped mouse, Rhabdomys.

Kikusui, T., Kiyokawa, Y., Mori, Y., 2007. Deprivation of mother-pup interaction by early weaning alters myelin formation in male, but not female, ICR mice. Brain Res. 1133, 115-122.

Kikusui, T., Nakamura, K., Mori, Y., 2008. A review of the behavioural and neurochemical consequences of early weaning in rodents. Appl. Anim. Behav. Sci. 110, 73-83.

König, B., Markl, H., 1987. Maternal care in house mice. Behav. Ecol. Sociobiol. 20, 1-9.

Latham, N., Mason, G.J., 2008. Maternal deprivation and the development of stereotypic behaviour. Appl. Anim. Behav. Sci. 110, 84-108.

Laviola, G., Terranova, M.L., 1998. The developmental psychobiology of behavioural plasticity in mice: the role of social experiences in the family unit. Neurosci. Biobehav. Rev. 23, 197-213.
Lewis, M.H., Presti, M.F., Lewis, J.B., Turner, C.A., 2006. The neurobiology of stereotypy I: environmental complexity. In: Mason, G., Rushen, J. (Eds.), Stereotypic Animal Behaviour: Fundamentals and Applications to Welfare. second ed. CAB International, Oxford, pp. 190-226.

Mason, G.J., 1995. Tail-biting in mink (Mustela vison) is influenced by age at removal from the mother. Anim. Welf. 3, 305-311.

Mason, G.J., Latham, N., 2004. Can't stop, won't stop: is stereotypy a reliable animal welfare indicator? Anim. Welf. 13, S57-S69.

Nel, K.N., 2003. The effects of dietary protein on the reproduction and behavioural characteristics of the striped mouse, Rhabdomys pumilio. MSc thesis, University of the Witwatersrand.

Newberry, R.C., Swanson, J.C., 2008. Implications of breaking motheryoung social bonds. Appl. Anim. Behav. Sci. 110, 3-23.

Pillay, N., 2000. Female mate preference and reproductive isolation in populations of the striped mouse Rhabdomys pumilio. Behaviour 137, $1431-1441$.

Rymer, T., 2009. Paternal care in the striped mouse Rhabdomys pumilio: ontogeny and function. PhD thesis, University of the Witwatersrand.

Schradin, C., Pillay, N., 2003. The influence of the father on offspring development in the striped mouse. Behav. Ecol. 16, 450-455.

Schwaibold, U., Pillay, N., 2001. Stereotypic behaviour is genetically transmitted in the African striped mouse Rhabdomys pumilio. Appl. Anim. Behav. Sci. 74, 273-280.

StatSoft, Inc., 2007. STATISTICA (data analysis software system), version 8.0. www.statsoft.com

Willan, K., Meester, J., 1989. Life-history styles of southern African Mastomys natalensis, Otymys irroratus and Rhabdomys pumilio (Mammalia, Rodentia). In: Bruton, M.N. (Ed.), Alternative Life-History Styles of Animals. Kluwer Academic Publishers, Dordrecht, pp. 421-439.

Wright, S.L., Brown, R.E., 2002. The importance of paternal care on pup survival and pup growth in Peromyscus californicus when required to work for food. Behav. Proc. 60, 41-52.

Würbel, H., Stauffacher, M., 1997. Age and weight at weaning affect corticosterone level and development of stereotypies in ICR-mice. Anim. Behav. 53, 891-900.

Zar, J.H., 1996. Biostatistical Analysis, third ed. Prentice Hall, London. 